\title{
O GÊNERO TRACHELOMONAS (EUGLENOPHYCEAE) DE DUAS ÁREAS DOS MARIMBUS (BAIANO E REMANSO), CHAPADA DIAMANTINA, BAHIA, BRASIL.
}

\author{
$\underline{\text { Adones de Jesus Santos Pereira }}{ }^{1}$; Carlos Wallace do Nascimento Moura ${ }^{2}$ \\ 1. Bolsista PIBIC/FAPESB, Graduando em Bacharelado em Ciências Biológicas, Universidade Estadual de Feira de \\ Santana, e-mail: adones2006@gmail.com \\ 2. Orientador, Departamento de Ciências Biológicas, Universidade Estadual de Feira de Santana, e-mail: \\ wallace@uefs.br
}

PALAVRAS-CHAVE: Microalga; Taxonomia; Inventário.

\section{INTRODUÇÃO}

A divisão Euglenophyta é uma das muitas divisões de microalgas existentes, e atualmente engloba 40 gêneros e aproximadamente 800 espécies (Parra \& Bicudo, 1995), embora segundo Norton et al. (1996) há estimativa de duas mil espécies pertencentes à divisão.

Trachelomonas Ehrenberg emend. Deflandre é o gênero mais representativo da divisão Euglenophyta, com cerca de 260 espécies (Alves-da-Silva 2007), habitando ambientes de água doce (Bourrelly, 1970), bem como o marinho (Leedale, 1967).

O gênero inclui algas de vida livre e hábito solitário, sendo caracterizado por apresentar célula com um envelope rígido e mucilaginoso denominado de lórica, que pode apresentar diferentes formatos e padrões de ornamentação e coloração (Tell \& Conforti, 1986), os quais são utilizados na taxonomia do grupo. A lórica é impregnada por depósitos de compostos férricos e mangânicos (Leedale, 1975; Dunlap et al., 1983) e apresenta um poro no pólo anterior da célula através do qual emerge o flagelo.

Trabalhos taxonômicos de microalgas no estado do Bahia ainda são escassos, diante da complexidade de suas bacias de drenagem e da diversidade de ecossistemas aquáticos continentais. Com relação ao gênero Trachelomonas, todas as informações disponíveis estão restritas a checklists (Fuentes et al., 2010; Santana, 2011; Severiano et al., 2012; Alves-da-Silva \& Menezes, 2015).

Diante do exposto, o presente trabalho teve como objetivo a realização de um estudo taxonômico das Trachelomonas ocorrentes em duas áreas dos Marimbus (Baiano e Remanso), Chapada Diamantina, Bahia, Brasil, de modo a ampliar o registro de espécies para a Bahia e, consequentemente, para o Brasil.

\section{MATERIAL E MÉTODOS}

O material é proveniente de coletas realizadas no período de abril de 2011 a fevereiro de 2012, em duas áreas do Pantanal dos Marimbus (Baiano e Remanso), totalizando 120 unidades amostrais, sendo 60 amostras obtidas no Marimbus do Baiano e 60 no Marimbus do Remanso; as encontram-se tombadas no Herbário HUEFS.

As amostras foram analisadas com auxílio do microscópio óptico binocular marca Olympus (modelo BX43) e as fotomicrografias obtidas com máquina fotográfica digital (Modelo QImaging 5.0) acoplado ao microscópio.

As dimensões dos espécimes foram aferidas através de ocular micrométrica. Aproximadamente 540 alíquotas foram preparadas das unidades amostrais e analisadas. A identificação taxonômica foi, sempre que possível, em nível infragenérico, 
analisando-se as características morfológicas e métricas das populações. Estas foram baseadas em trabalhos especializados, incluindo floras, revisões, dissertações e teses.

O sistema de classificação utilizado segue Guiry \& Guiry (2016).

\section{RESULTADOS E DISCUSSÃO}

Das 120 unidades amostrais analisadas, 94 apresentaram representantes de Trachelomonas, sendo 51 unidades no Marimbus do Baiano e 43 no Marimbus do Remanso.

O gênero Trachelomonas foi representado na flora local por 58 táxons, sendo 34 identificados a nível infraespecífico (21 espécies, 13 variedades que não as típicas de suas respectivas espécies, uma forma taxonômica não típica, porém, pertencente à variedade típica de sua espécie [Trachelomonas armata var. armata f. inevoluta] e uma forma taxonômica não típica, pertencente a uma variedade igualmente não típica de sua espécie [Trachelomonas raciborskii var. nova f. minor]), cinco variedades não identificadas pertencentes a três espécies e 19 táxons identificados a nível de gênero.

O Marimbus do Baiano apresentou maior riqueza de táxons (51, sendo 24 exclusivos) quando comparado ao Marimbus do Remanso (34, dos quais sete foram exclusivos).

Trachelomonas kellogii var. kellogii foi o táxon mais representativo, tanto no Marimbus do Baiano quanto no Remanso, tendo sido registrado em 56 unidades amostrais.

Tabela. 1. Listagem dos táxons de Trachelomonas (Euglenophyceae) anteriormente referidas para o Estado e dos dados obtidos nos Marimbus do Baiano e do Remanso, Chapada Diamantina, Bahia, Brasil. (1- Fuentes et al. (2010), 2- Santana (2011), 3- Severiano et al. (2012), 4 - Alves-da-Silva \& Menezes (2015), BR- novo registro para o Brasil, NE- Novo registro para o Nordeste do Brasil, BA- novo registro para a Bahia, MB- ocorrência registrada no Marimbus do Baiano, MR - ocorrência registrada no Marimbus do Remanso).

\begin{tabular}{|c|c|c|c|c|c|c|}
\hline \multirow[b]{2}{*}{ Táxons } & \multirow{2}{*}{$\begin{array}{c}\text { Citações } \\
\text { anteriores }\end{array}$} & \multicolumn{5}{|c|}{ Presente trabalho } \\
\hline & & BA & NE & BR & MB & MR \\
\hline T. abrupta Swirenko emend. Deflandre var. abrupta & 2 & $\mathrm{x}$ & & & $\mathrm{x}$ & $\mathrm{x}$ \\
\hline T. abrupta Swirenko emend. Deflandre var. arcuata (Playfair) Deflandre & & & $\mathrm{x}$ & & $\mathrm{x}$ & $\mathrm{x}$ \\
\hline T. acanthophora Stokes & 2 & & & & & \\
\hline T. allia Drezepolski emend. Deflandre var. obesa Balech & & & & $\mathrm{x}$ & $\mathrm{x}$ & $\mathrm{x}$ \\
\hline T. armata (Ehrenberg) F.Stein var. armata f. armata & 2,3 & & $\mathrm{x}$ & & $\mathrm{x}$ & $\mathrm{x}$ \\
\hline T. armata (Ehrenberg) F.Stein var. armata f. inevoluta Deflandre & & & $\mathrm{x}$ & & $\mathrm{x}$ & $\mathrm{x}$ \\
\hline T. armata (Ehrenberg) F.Stein var. litoralensis Tell \& Domitrovic & 2 & & $\mathrm{x}$ & & $\mathrm{x}$ & \\
\hline T. armata (Ehrenberg) F.Stein var. longispina Playfair emend. Deflandre & 2 & $\mathrm{x}$ & & & $\mathrm{x}$ & $\mathrm{x}$ \\
\hline T. armata var. ovata Swirenko & 2 & & & & & \\
\hline T. armata (Ehrenberg) F.Stein var. sparsigranosa Playfair & & & $\mathrm{x}$ & & $\mathrm{x}$ & \\
\hline T. armata (Ehrenberg) F.Stein var. steinii Lemmermann emend. Deflandre & 2 & & $\mathrm{x}$ & & $\mathrm{x}$ & $\mathrm{x}$ \\
\hline T. armata var. 1 & & & & & $\mathrm{x}$ & \\
\hline T. armata var. 2 & & & & & $\mathrm{x}$ & \\
\hline T. armata var. 3 & & & & & $\mathrm{x}$ & \\
\hline T. balechii Tell & & & $\mathrm{x}$ & & $\mathrm{x}$ & \\
\hline T. bulla F.Stein emend. Deflandre. var. bulla & 2 & & & & & \\
\hline T. conica Playfair & & & $\mathrm{x}$ & & $\mathrm{x}$ & $\mathrm{x}$ \\
\hline T. curta A.M.Cunha emend. Deflandre var. curta & & $\mathrm{x}$ & & & $\mathrm{x}$ & $\mathrm{x}$ \\
\hline T. curta A.M.Cunha emend. Deflandre var. subpunctata Bourrelly & & & & $\mathrm{x}$ & & $\mathrm{x}$ \\
\hline T. dangeardiana (Deflandre) Huber-Pestalozzi var. glabra (Playfair) Deflandre & & & $\mathrm{x}$ & & & $\mathrm{x}$ \\
\hline T. dastuguei Balech var. dastuguei f. dastuguei & 2 & & & & & \\
\hline T. hemispherica Garcia-de-Emiliani & 2 & & & & & \\
\hline T. hirta A.M.Cunha var.hirta & 2 & & & & & \\
\hline T. hispida (Perty) F.Stein emend. Deflandre var. hispida & 2 & & $\mathrm{x}$ & & $\mathrm{x}$ & $\mathrm{x}$ \\
\hline T. hispida (Perty) F.Stein emend. Deflandre var. coronata Lemmermann & & $\mathrm{x}$ & & & $\mathrm{x}$ & $\mathrm{x}$ \\
\hline T. hispida (Perty) F.Stein emend. Deflandre var. duplex Deflandre & & & $\mathrm{x}$ & & $\mathrm{x}$ & $\mathrm{x}$ \\
\hline T. hispida (Perty) F.Stein emend. Deflandre var. punctata Lemmermann & & & $\mathrm{x}$ & & $\mathrm{x}$ & \\
\hline T. intermedia P.A.Dangeard var. intermedia & & & $\mathrm{x}$ & & $\mathrm{x}$ & $\mathrm{x}$ \\
\hline T. intermedia P.A.Dangeard var. minor Tell & & & $\mathrm{x}$ & & $\mathrm{x}$ & $\mathrm{x}$ \\
\hline T. kellogii Skvortzov emend. Deflandre var. kellogii & & & $\mathrm{x}$ & & $\mathrm{x}$ & $\mathrm{x}$ \\
\hline T. lacustris Drezepolski var. lacustris & 2 & & $\mathrm{x}$ & & $\mathrm{x}$ & $\mathrm{x}$ \\
\hline T. lemmermannii Woloszýnska emend. Deflandrevar. lemmermannii & & & $\mathrm{x}$ & & $\mathrm{x}$ & $\mathrm{x}$ \\
\hline T. lemmermannii Woloszýnska var.acuminata Deflandre & 2 & & & & & \\
\hline T. megalacantha A.M.Cunha var. megalacantha & & & $\mathrm{x}$ & & $\mathrm{x}$ & \\
\hline T. obesa Ehrenberg & 1,3 & & & & & \\
\hline T. oblong a Lemmermann var. oblonga & 1,3 & & $\mathrm{x}$ & & $\mathrm{x}$ & $\mathrm{x}$ \\
\hline T. parvicollis Deflandre & 2 & & & & & \\
\hline T. raciborskii Woloszýnska var. raciborskii & 2 & $\mathrm{x}$ & & & $\mathrm{x}$ & $\mathrm{x}$ \\
\hline T. raciborskii Woloszýnska var. nova Drezepolski & & & $\mathrm{x}$ & & & $\mathrm{x}$ \\
\hline
\end{tabular}




\begin{tabular}{|c|c|c|c|c|c|c|}
\hline \multirow{2}{*}{ Táxons } & \multirow{2}{*}{$\begin{array}{c}\text { Citações } \\
\text { anteriores }\end{array}$} & \multicolumn{5}{|c|}{ Presente trabalho } \\
\hline & & BA & NE & BR & MB & MR \\
\hline T. raciborskii Woloszýnska var. nova Drezepolski f. minor T. Hortobágyi & & & & $\mathrm{x}$ & $\mathrm{x}$ & $\mathrm{x}$ \\
\hline T. robusta Swirenko var. robusta & & & $\mathrm{x}$ & & $\mathrm{x}$ & $\mathrm{x}$ \\
\hline T. rugulosa F.Stein var. rugulosa & & & $\mathrm{x}$ & & $\mathrm{x}$ & \\
\hline T. sculpta Balech var. sculpta & & & $\mathrm{x}$ & & $\mathrm{x}$ & \\
\hline T. similis Stokes & 2 & & & & & \\
\hline T. superba Swirenko emend. Deflandre & 2 & & $\mathrm{x}$ & & $\mathrm{x}$ & $\mathrm{x}$ \\
\hline T. volvocina (Ehrenberg) Ehrenberg var. volvocina & $1,2,3,4$ & $\mathrm{x}$ & & & $\mathrm{x}$ & $\mathrm{x}$ \\
\hline T. volvocina Ehrenberg var. compressa Deflandre & 2 & & & & & \\
\hline T. volvocina var. & & & & & $\mathrm{x}$ & \\
\hline T. volvocinopsis Swirenko var. volvocinopsis & & & $\mathrm{x}$ & & & $\mathrm{x}$ \\
\hline T. volvocinopsis var. & & & & & $\mathrm{x}$ & $\mathrm{x}$ \\
\hline Trachelomonas sp. 1 & & & & & $\mathrm{x}$ & $\mathrm{x}$ \\
\hline Trachelomonas sp. 2 & & & & & $\mathrm{x}$ & $\mathrm{x}$ \\
\hline Trachelomonas sp. 3 & & & & & $\mathrm{x}$ & $\mathrm{x}$ \\
\hline Trachelomonas sp. 4 & & & & & & $\mathrm{x}$ \\
\hline Trachelomonas sp. 5 & & & & & & $\mathrm{x}$ \\
\hline Trachelomonas sp. 6 & & & & & $\mathrm{x}$ & \\
\hline Trachelomonas sp. 7 & & & & & $\mathrm{x}$ & \\
\hline Trachelomonas $\mathrm{sp} .8$ & & & & & $\mathrm{x}$ & \\
\hline Trachelomonas sp. 9 & & & & & $\mathrm{x}$ & \\
\hline Trachelomonas sp. 10 & & & & & $\mathrm{x}$ & \\
\hline Trachelomonas sp. 11 & & & & & $\mathrm{x}$ & \\
\hline Trachelomonas sp. 12 & & & & & $\mathrm{x}$ & \\
\hline Trachelomonas sp. 13 & & & & & & $\mathrm{x}$ \\
\hline Trachelomonas sp. 14 & & & & & $\mathrm{x}$ & \\
\hline Trachelomonas sp. 15 & & & & & $\mathrm{x}$ & \\
\hline Trachelomonas sp. 16 & & & & & $\mathrm{x}$ & \\
\hline Trachelomonas sp. 17 & & & & & $\mathrm{x}$ & \\
\hline Trachelomonas sp. 18 & & & & & $\mathrm{x}$ & \\
\hline Trachelomonas sp. 19 & & & & & $\mathrm{x}$ & \\
\hline
\end{tabular}

\section{CONCLUSÕES}

O estudo das 120 unidades amostrais coletadas em duas áreas do Pantanal dos Marimbus (Baiano e Remanso), Chapada Diamantina, permitiu concluir que:

- A área apresentou expressiva biodiversidade - 58 táxons dos quais, três constituem novos registros para o Brasil ( $T$. allia var. obesa, $T$. curta var. subpunctata, $T$. raciborskii var. nova $\mathrm{f}$. minor);

- 25 táxons de Trachelomonas foram adicionados à flora ficológica do Nordeste do Brasil (T. abrupta var. arcuata, T. armata var. armata f. armata, T. armata var. sparsigranosa, T. armata var. armata f. inevoluta, T. armata var. litoralensis, T. armata var. steinii, T. balechii, T. conica, T. dangeardiana var. glabra, T. hispida var. hispida, T. hispida var. duplex, T. hispida var. punctata, T. intermedia var. intermedia, $T$. intermedia var. minor, $T$. kellogii var. kellogii, $T$. lacustris var. lacustris, $T$. lemmermannii var. lemmermannii, T. megalacantha var. megalacantha, T. oblonga var. oblonga, T. raciborskii var. nova, T. robusta var. robusta, T. rugulosa var. rugulosa, $T$. sculpta var. sculpta, T. superba, T. volvocinopsis var. volvocinopsis);

- Seis táxons constituem novos registros para o estado da Bahia: (T. abrupta var. abrupta, T. armata var. longispina, T. curta var. curta, T. hispida var. coronata, T. raciborskii var. raciborskii, T. volvocina var. volvocina);

- 19 táxons (Trachelomonas sp. 1 a sp. 19) não puderam ser encaixados em quaisquer circunscrições de Trachelomonas já descritas e, muito provavelmente, constituem-se novidades para a ciência;

- Cinco variedades são prováveis táxons novos para a ciência (T. armata var. $1, T$. armata var. 2, T. armata var. $3, T$. volvocina var. e $T$. volvocinopsis var.); 


\section{REFERÊNCIAS}

PARRA, O.O.; BICUDO, C.E.M. 1995. Introdução a la biología y sistemática de las algas continentales. Chile: Universidad de Concepción.

NORTON, T.A.; MELKONIAN, M.; ANDERSEN, R.A. 1996. Algal biodiversity. Phycologia 35: 308-326.

ALVES-DA-SILVA, SM. 2007. Primeiro registro de Trachelomonas amphoriformis var. granulosa (Euglenophyceae) no Rio Grande do Sul, Brasil. Revista Brasileira de Biociências 5: 522-524.

BOURRELLY, P.C. 1970. Les algues d'eau douce: initiation à la systématique. Tome III. Les algues blues et rouges. Les Eugléniens, Peridiniens et Cryptomonadines. Paris: Éditions N. Boubée.

LEEDALE, G.F. 1967. Euglenoid Flagellates. Englewood, New Jersey: Prentice Hall.

TELL, G.; CONFORTI, V. 1986. Euglenophyta Pigmentadas de la Argentina. Bibliotheca Phicologica 75: 1-301.

FUENTES, E.V.; OLIVEIRA, H.S.B.; CORDEIRO-ARAÚJO, M.K.; SEVERI, W.; MOURA, A.N. 2010. Variação espacial e temporal do fitoplâncton do Rio de Contas, Bahia, Brasil. Revista Brasileira de Engenharia de Pesca 5(2): 13-25.

SANTANA, L.M. 2011. Análise física e química da água e estrutura da comunidade fitoplanctônica do rio Almada (sul da Bahia). Dissertação de Mestrado, Programa de Pós-Graduação em Sistemas Aquáticos Tropicais, Universidade Estadual de Santa Cruz, Bahia.

SEVERIANO, J.S.; MOURA, A.N.; BEZERRA-OLIVEIRA, H.S.; CORDEIROARAÚJO, M.K.; DANTAS, E.W. 2012. Microphytoplankton richness in Contas River, state of Bahia, northeastern Brazil. Checklist: Journal of Species Lists and Distribution 8(2): 218-223.

ALVES-DA-SILVA, S.M.; MENEZES, M. 2015. Euglenophyceae in Lista de Espécies da Flora do Brasil. Jardim Botânico do Rio de Janeiro. Disponivel em: <http://floradobrasil.jbrj.gov.br/jabot/floradobrasil/FB109138>.

FUNCH, R. 1997. Um guia para o visitante da Chapada Diamantina: o circuito do diamante. Coleção Apoio, no 15. Salvador: Empresa Gráfica da Bahia.

BICUDO, C.E.M.; MENEZES, M. 2006. Gêneros de algas de águas continentais do Brasil: chave para identificação e descrições. 2 ed. Rima, São Carlos.

GUIRY in GUIRY, M.D.; GUIRY, G.M. 2016. AlgaeBase. World-wide electronic publication, National University of Ireland, Galway. http://www.algaebase.org. Acesso 3 Mai 2016. 